CARDIOVASCULAR MEDICINE

\title{
Is echocardiography a valid tool to screen for left ventricular systolic dysfunction in chronic survivors of acute myocardial infarction? A comparison with radionuclide ventriculography
}

\author{
G I W Galasko, S Basu, A Lahiri, R Senior
}

Heart 2004;90:1422-1426. doi: 10.1136/hrt.2003.027425

See end of article for authors' affiliations

.....................

Correspondence to: Dr Roxy Senior, Department of

Cardiovascular Medicine Northwick Park Hospital, Harrow, Middlesex HAl 3UJ, UK; roxy.senior@ virgin.net

Accepted 7 February 2004

\begin{abstract}
Objective: To assess the accuracy of echocardiography with Simpson's apical biplane method in screening for left ventricular systolic dysfunction (LVSD) in patients six months after acute myocardial infarction (AMI) as compared with radionuclide ventriculography by assessing the proportion of clinically significant errors that occur with echocardiography.

Design: Comparison of results of echocardiography and radionuclide ventriculography in assessing left ventricular ejection fraction among patients six months after AMI.

Setting: District general hospital.

Patients: 86 patients thrombolysed for AMI at six month follow up.

Interventions: None.

Main outcome measures: Correlation coefficients, mean differences, 95\% limits of agreement, and differences of clinical significance between left ventricular ejection fraction on echocardiography and on radionuclide ventriculography.

Results: The correlation coefficient between techniques was 0.90 , mean difference $1 \%(p=0.04)$, and $95 \%$ limits of agreement $-13.0 \%$ to $10.3 \%$. Only one patient $(1.2 \%, 0.0 \%$ to $6.3 \%)$ was classified as having normal systolic function on one imaging modality but significant LVSD on the other. Overall accuracy between the two techniques was $86 \%$, $\kappa$ value of agreement 0.78 .

Conclusion: Echocardiography is a valid tool to screen for LVSD in patients six months after AMI, accurately differentiating normal from abnormal systolic function and showing excellent agreement with radionuclide ventriculography. This study supports the use of echocardiography in screening for LVSD in chronic stable patients after AMI or alternative high risk patients, with few differences of major clinical significance likely to occur.
\end{abstract}

$\mathrm{H}$ eart failure is one of the most common chronic diseases of the western world with high associated morbidity, mortality, and cost. ${ }^{1}$ Left ventricular systolic dysfunction (LVSD) underlies most heart failure cases but is often asymptomatic before the development of clinical heart failure. $^{2-4}$ If drug treatment is initiated during this latent asymptomatic stage, then the development of chronic heart failure and its consequences can be delayed if not prevented. ${ }^{4}$ If drug treatment is initiated once symptoms have developed then morbidity and mortality can be greatly reduced. ${ }^{5}$ Unfortunately, symptomatic LVSD is commonly misdiagnosed, especially in primary care where facilities may be limited, ${ }^{6}$ leading authors to call for programmes to screen for and treat both symptomatic and asymptomatic LVSD. ${ }^{2}$

As in all screening programmes, a safe, reliable, and accurate screening method is required. Echocardiography has been proposed to be the screening method of choice, ${ }^{78}$ although no study thus far has validated its use in this setting-assessing the proportion of clinically significant errors that occur when using echocardiography to screen for LVSD compared with an alternative imaging modality. Left ventricular ejection fraction (LVEF) is the most common measure of left ventricular systolic function, with Simpson's apical biplane method (the biplane method of discs) recommended as the most accurate echocardiographic measure of LVEF, having the fewest geometric assumptions. Indeed, several community studies have used Simpson's apical biplane method to detect LVSD but none has compared its results against an alternative measure of LVSD. ${ }^{10-12} \mathrm{~A}$ validation study is especially necessary, as some studies have cast doubt on the reliability of Simpson's apical biplane method in detecting LVSD, especially acutely or in the chronic phase after acute myocardial infarction (AMI), ${ }_{13} 14$ where regional wall motion abnormalities often occur. ${ }^{15-17}$ As prior AMI is the most common cause of asymptomatic LVSD in the community, ${ }^{4}{ }^{10}{ }^{11}$ echocardiography would need to be shown to screen accurately for LVSD in such patients before it would be accepted as a valid screening tool.

Accordingly, this study was undertaken to assess the accuracy of echocardiography with Simpson's apical biplane method in detecting LVSD in patients in the chronic phase after AMI. We assessed the proportion of clinically significant errors that occur with this technique. Radionuclide ventriculography was used as a validated reproducible comparative measure of LVEF in this analysis. ${ }^{18-20}$

\section{METHODS}

\section{Patients}

Data were analysed from the six month follow up data of the CHAPS (carvedilol heart attack pilot study), a randomised double blind study of carvedilol or placebo in patients after

Abbreviations: AMI, acute myocardial infarction; CHAPS, carvedilol heart attack pilot study; LVEF, left ventricular ejection fraction; LVSD, left ventricular systolic dysfunction; SAVE, survival and ventricular enlargement; WMSI, wall motion score index 
AMI. ${ }^{21}$ Eighty six patients underwent both echocardiography and radionuclide ventriculography on the same day at six months' follow up. ${ }^{16}$

\section{Radionuclide ventriculography}

Red blood cells were labelled in vivo by an intravenous injection of $10 \mathrm{mg}$ stannous pyrophosphate followed by an injection of $740 \mathrm{MBq}$ of technetium-99m 30 minutes later. Multiple gated radionuclide ventriculograms were obtained by means of a gamma camera with a low energy collimator (Apex $215 \mathrm{M}$, Elscint). The camera was positioned in the $30^{\circ}-45^{\circ}$ left anterior oblique projection with a $5^{\circ}-10^{\circ}$ caudal tilt to isolate the left ventricle. The RR interval was divided into 32 frames and a 5\% gate tolerance was used. Five million counts were collected and the data stored in a $64 \times 64$ matrix to obtain a high resolution time-activity curve of the change in precordial radioactivity. The LVEF was obtained in the left anterior oblique view by a second derivative semiautomatic edge detection programme, as previously described by our laboratory ${ }^{22}$ and reported by one observer $(\mathrm{AL})$, who was blinded to the echocardiography results. The interobserver and intraobserver variability in measuring radionuclide ventriculography LVEF after AMI in our laboratory has previously been published. ${ }^{23}$

\section{Echocardiography}

Two dimensional echocardiography was performed by one investigator (RS) using a phased array ultrasound imaging system with a $2.25 \mathrm{MHz}$ transducer (Ultramark 9, ATL). RS was blinded to the radionuclide ventriculography results. LVEF was calculated in the apical two and four chamber views with Simpson's apical biplane method. ${ }^{24}$ All studies were recorded on VHS videotape and data were analysed off line with the Ultramark 9 in-built computer system. The stop frame model and the reference ECG were used to identify the left ventricular end diastolic endocardial borders at the peak of the $\mathrm{R}$ wave. The end systolic endocardial borders were measured near the end of the $\mathrm{T}$ wave at the maximum inward motion of the left ventricle. In tracing the cross sectional echocardiographic endocardial borders, minor irregularities due to decreased visual integrity of the endocardium were interpolated from the real time and slow motion images. An average of the ejection fraction was obtained from three cardiac cycles avoiding any extrasystolic or post-extrasystolic cycles. The interobserver and intraobserver variability in measuring LVEF after AMI with this technique in our laboratory has previously been published. ${ }^{23}$

Global systolic wall motion score index (WMSI) was analysed by an 11 segment model ${ }^{16}$ with six segments each assigned to anterior and inferior regions, the apex being common. The motion of individual segments was graded as follows: normal, 0; hypokinesia, 1; akinesia, 2; and dyskinesia, 3. Global systolic wall motion score was calculated by dividing the total score by the number of segments analysable. Results were considered only when at least four segments from each of the anterior and inferior regions were analysable. The intraobserver variability of measuring WMSI after AMI in our laboratory has previously been published. ${ }^{25}$

\section{Statistical analysis}

Binomial confidence intervals (95\%) were used for prevalence rates. Echocardiographic LVEF and radionuclide ventriculography LVEF were compared by paired sample $t$ tests, Pearson's correlation coefficients, and Bland-Altman 95\% limits of agreements. Significant LVSD was defined as LVEF $<40 \%$, borderline LVSD as LVEF $40-50 \%$, and normal systolic function as LVEF $>50 \%$, according to previous epidemiological studies. ${ }^{1126}$ The overall accuracy and $\kappa$ value of agreement $(<0.4$ poor, $0.4-0.75$ good, and $>0.75$ excellent agreement ${ }^{27}$ ) for echocardiography to predict LVSD were assessed. Differences of major clinical significance were defined as LVEF $>50 \%$ on one modality but $<40 \%$ on the other. Data were analysed by Analyse-it for Excel version 1.48 (Microsoft, Leeds, UK) and SPSS version 10.0.5 (SPSS Inc, Chicago, Illinois, USA).

\section{RESULTS}

\section{Patients}

Eighty six patients attended, 72 men and 14 women, mean (SD) age 60.5 (10.5) years (range $34-80$ years). Forty (47\%) had presented with an anterior AMI and 46 (53\%) with an inferior AMI six months earlier. Fifty seven (66\%) were white, 28 (33\%) South Asian, and one (1\%) Afro-Caribbean.

\section{Wall motion score index}

WMSI was successfully assessed in 85 of the 86 patients at their six month follow up visit. The overall mean WMSI was 0.5 (0.5). Twenty patients $(24 \%)$ had no wall motion abnormality, 27 (32\%) had one to three abnormally contracting walls, and 38 (45\%) had four or more abnormally contracting walls. Thirty three patients $(39 \%)$ had a WMSI $\geqslant 0.6$, previously shown to equate to $\mathrm{LVEF} \leqslant 45 \%$. $^{16}$

\section{Comparison between radionuclide ventriculography and echocardiographic ejection fraction}

LVEF was calculable in all 86 cases by both techniques. Overall mean LVEFs for radionuclide ventriculography and echocardiography, respectively, were $45 \%$ versus $44 \%$
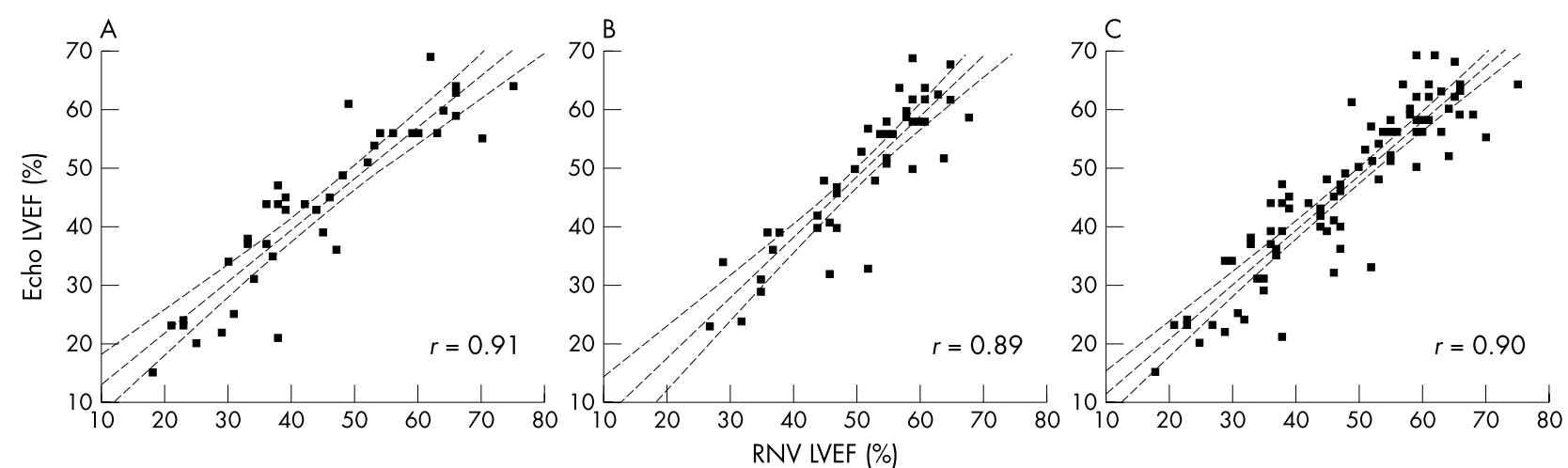

Figure 1 Scatter plots of left ventricular ejection fraction by radionuclide ventriculography (RNV LVEF) and by echocardiography (Echo LVEF) (A) in 40 patients six months after anterior myocardial infarction, (B) in 46 patients six months after inferior myocardial infarction, and (C) in all 86 patients combined. Regression lines and $95 \%$ confidence intervals for the regression lines are plotted. 



Figure 2 Bland-Altman limits of agreement plots between RNV LVEF and Echo LVEF (A) in 40 patients six months after anterior myocardial infarction, $(B)$ in 46 patients six months after inferior myocardial infarction, and (C) in all 86 patients combined.

$(\mathrm{p}=0.27)$ for anterior AMIs, $51 \%$ versus $50 \%(\mathrm{p}=0.06)$ for inferior AMIs, and 48\% versus $47 \%(\mathrm{p}=0.04)$ for all patients combined.

Figure 1 depicts scatter plots showing the correlation between radionuclide ventriculography and echocardiographic LVEF, with correlation coefficients given. Figure 2 shows Bland-Altman limits of agreement plots and table 1 gives the $95 \%$ limits of agreement.

\section{Differences of clinical significance}

Table 2 lists the differences of clinical significance between echocardiographic and radionuclide ventriculographic determination of LVEF. The overall accuracy of echocardiography in correctly assessing radionuclide ventriculography LVEF was $86 \%$ ( $\kappa$ value of agreement 0.78 ), suggesting excellent agreement. Only one patient (accuracy 1.2\%, 95\% limits of agreement $0.0 \%$ to $6.3 \%$ ) was found to have a difference of major clinical significance. This patient, having had a prior inferior AMI, was found to have significant LVSD on echocardiography but normal systolic function on radionuclide ventriculography. No patients were found to have significant LVSD on radionuclide ventriculography but normal systolic function on echocardiography. Eleven patients $(13 \%)$ had minor differences in classification. Five patients were classified as having significant LVSD on radionuclide ventriculography but only borderline LVSD on echocardiography. One patient was classified as having borderline LVSD on radionuclide ventriculography but normal systolic function on echocardiography. All six patients had had anterior AMIs. Similarly, three patients were classified as having significant LVSD on echocardiography but only borderline LVSD on radionuclide ventriculography, and two patients were classified as having borderline LVSD on echocardiography but normal systolic function on

Table 1 Mean difference and $95 \%$ limits of agreement between echocardiography (Echo) and radionuclide ventriculography (RNV) assessment of left ventricular ejection fraction (LVEF) after acute myocardial infarction (AMI)

\begin{tabular}{lllll}
\hline AMI & $\begin{array}{l}\text { Mean difference } \\
\text { (Echo-RNV) }\end{array}$ & $\begin{array}{l}\text { Number of } \\
\text { patients }\end{array}$ & $\begin{array}{l}\text { 95\% Limits of } \\
\text { agreement }\end{array}$ & p Value \\
\hline All & -1.3 & 86 & -13.0 to 10.3 & 0.04 \\
Anterior & -1.1 & 40 & -13.4 to 11.2 & 0.27 \\
Inferior & -1.5 & 46 & -12.2 to 9.1 & 0.06 \\
\hline
\end{tabular}

radionuclide ventriculography. Three of these five patients had had inferior AMIs.

\section{DISCUSSION}

This study has found very good agreement between echocardiography and radionuclide ventriculography in assessing left ventricular systolic function in patients six months after AMI. This is despite a large range of LVEFs recorded (18-75\% by radionuclide ventriculography and 15$69 \%$ by echocardiography) and a large proportion of patients with significant wall motion abnormalities. Overall there was a significant difference between the two techniques of only $1 \%$. LVEF was successfully calculated for all patients with Simpson's apical biplane method. There was $86 \%$ agreement between the two techniques, with an excellent $\kappa$ value of agreement. Only one of 86 patients was classified as having significant LVSD on echocardiography but normal systolic function on radionuclide ventriculography, and no patients were classified as having normal systolic function on echocardiography but significant LVSD on radionuclide ventriculography. This implies minimal differences of major clinical significance between the two techniques. This was despite $95 \%$ limits of agreement between the two techniques of $-13.0 \%$ to $10.3 \%$.

Twelve patients (14\%), however, were misclassified. Of the 40 patients with normal systolic function on echocardiography, one $(2.5 \%)$ had borderline LVSD and none had significant LVSD on radionuclide ventriculography (negative predictive value $97.5 \%$ in ruling out any LVSD). This suggests that a normal echocardiography study accurately excludes LVSD, a necessary finding for any screening programme. Of the 26 patients with definite LVSD on echocardiography, three ( $12 \%$ ) had borderline LVSD and one (4\%) had normal systolic function on radionuclide ventriculography. This implies a positive predictive value of $85 \%$ in diagnosing significant LVSD and a positive predictive value of $96 \%$ in

Table 2 Agreement between RNV and echocardiography (Echo) in assessing LVEF in 86 patients six months after first presentation with AMI

\begin{tabular}{lcccc}
\hline \multirow{5}{*}{ Echo LVEF } & \multicolumn{2}{l}{ RNV LVEF } & & \\
\cline { 2 - 5 } & $<\mathbf{4 0} \%$ & $\mathbf{4 0 - 5 0 \%}$ & $>\mathbf{5 0} \%$ & Total \\
\hline$<40 \%$ & $\mathbf{2 2}$ & 3 & 1 & 26 \\
$40-50 \%$ & 5 & 13 & 2 & 20 \\
$>50 \%$ & 0 & 1 & 39 & 40 \\
Total & 27 & 17 & 42 & 86 \\
\hline
\end{tabular}


diagnosing at least borderline LVSD, with high positive predictive value also a vital component of any screening programme. Of the 20 patients with borderline LVSD on echocardiography, five (25\%) had significant LVSD and two $(10 \%)$ had normal systolic function on radionuclide ventriculography. Thus, although borderline LVSD on echocardiography gave a positive predictive value of $90 \%$ in diagnosing at least borderline LVSD on radionuclide ventriculography, over a third of patients were partially misclassified, some having normal systolic function. It may be necessary, therefore, for patients with borderline LVSD on echocardiography to undergo an alternative assessment of LVSD such as radionuclide ventriculography, magnetic resonance imaging, or contrast left ventriculography or simply repeat echocardiography at follow up to help assess disease severity. Such an approach has also previously been shown to help stratify prognosis, with a subgroup analysis of the SAVE (survival and ventricular enlargement) study finding that patients with abnormal LVEF on both radionuclide ventriculography and contrast left ventriculography have worse prognosis than those with abnormal results on one technique but normal LVEF on the other. ${ }^{28}$

These slight differences between echocardiography and radionuclide ventriculography may result from methodological differences between the two techniques, with radionuclide ventriculography relying on total ventricular counts to calculate LVEF. ${ }^{28}$ Thus, patients with large akinetic anterolateral walls or apices would contribute more to overall counts from these affected regions, underestimating LVEF, while patients with inferoposterior infarctions would contribute less to overall counts from these affected regions, overestimating LVEF. This hypothesis is supported by the current study, in which all six patients with LVEF overestimated by echocardiography were classified as having had anterior AMIs and four of the six patients with LVEF underestimated by echocardiography were classified as having had inferior AMIs. Furthermore, all six patients with LVEF overestimated by echocardiography had significant apical wall motion abnormalities; four patients also had significant anterior wall motion abnormalities. All six patients with LVEF underestimated by echocardiography had either significant inferior or posterior wall motion abnormalities; four patients had both abnormalities. Such a phenomenon, if true, is likely to arise in only a small subset of patients, however; neither regression line in fig $\mathrm{lA}$ or $\mathrm{B}$ differed significantly from the line of identity, implying no overall interaction between the infarct site and the relation between LVEF measured by echocardiography and by radionuclide ventriculography.

The overall results from the study are similar to results from previous studies of fewer patients. ${ }^{23}{ }^{30}$ Senior and colleagues $^{23}$ found $95 \%$ limits of agreement between radionuclide ventriculography and echocardiography in assessing LVEF of $-11.4 \%$ to $12.2 \%$ in 49 patients a few days after AMI. Similarly, Naik and colleagues ${ }^{30}$ found $95 \%$ limits of agreement of $-11.5 \%$ to $11.6 \%$ in 25 heterogeneous patients, 19 with prior AMI, two with dilated cardiomyopathy, and four with normal left ventricular function. Neither of these studies assessed differences of clinical significance between the two techniques.

Some studies have found worse agreement, however. ${ }^{13} 1431$ Bellenger and colleagues ${ }^{13}$ compared LVEF measured by magnetic resonance imaging versus by radionuclide ventriculography and echocardiography with Simpson's apical biplane method in 36 patients with heart failure and significant LVSD. Although they found no significant difference in mean LVEF between echocardiography and magnetic resonance imaging, they found 95\% limits of agreement of $-23.7 \%$ to $19.2 \%$. Similarly, when comparing echocardiography with radionuclide ventriculography in assessing LVEF, they found 95\% limits of agreement of $-26.7 \%$ to $12.0 \%$. All patients in that study had to have significant LVSD before study entry, however, and no patients with borderline LVSD or normal systolic function were entered into the study, unlike the current study. Indeed, with mean (SD) LVEFs of 31 (10)\% for echocardiography, 30 (11)\% for magnetic resonance imaging, and $24(9) \%$ for radionuclide ventriculography, it may be that very few if any attending patients were assessed as having normal systolic function on either technique. Thus, despite wide limits of agreement, it is likely that few, if any, differences of major clinical significance were seen. Ray and colleagues ${ }^{14}$ found similar results between LVEF measured by echocardiography with Simpson's apical biplane rule and LVEF measured by radionuclide ventriculography in 40 patients within 36 hours of AMI. ${ }^{14}$ They found a mean difference of $12 \%$ and $95 \%$ limits of agreement of $-35 \%$ to $8 \%$ between the two techniques. However, as in the previous study, their mean (2SD) values for LVEF were 33\% for radionuclide ventriculography and 50\% for echocardiography, so it is likely that few, if any, patients were assessed as having normal systolic function, also implying few, if any, differences of major clinical significance. A further criticism of that study is that, since the accuracy of Simpson's apical biplane method increases with the number of segments analysed, their use of only 10 segments for each analysis rather than the usual 20 segments, as used in the current study, may have reduced the accuracy of Simpson's apical biplane method. Jensen-Urstad and colleagues ${ }^{31}$ found similar poor agreement between echocardiographic LVEF with Simpson's apical biplane method and radionuclide ventriculography LVEF in 45 patients five to eight days after AMI, with 95\% limits of agreement of $\pm 19.6 \%$. However, there were potential methodological problems too. During echocardiography, LVEF was first estimated by visual inspection and then calculated quantitatively by Simpson's apical biplane method a few minutes later and the two values were compared. To avoid potential bias between these two methods, the study investigator was forbidden from redrawing the left ventricular cavity once the calculated volume or LVEF had been noted when using Simpson's method, even if an error had clearly been made. Unfortunately, doing this would have reduced the accuracy of the technique, potentially explaining the differences from the current study, where only optimal left ventricular cavity drawings were taken even if redrawing was required and the average of three consecutive measures was taken.

Naik and colleagues ${ }^{30}$ have shown that, despite wide limits of agreement occurring in some studies, differences of major clinical significance are still infrequent when comparing echocardiography with radionuclide ventriculography. They combined their data with data from seven other studies comparing LVEF by echocardiography and radionuclide ventriculography, for 174 patients in total. Although they did not formally test for differences of clinical significance, it can be calculated from presented data that only one patient $(0.5 \%)$ had LVEF $>50 \%$ on echocardiography but LVEF $<40 \%$ on radionuclide ventriculography, and no patients had LVEF $<40 \%$ on echocardiography but LVEF $>50 \%$ on radionuclide ventriculography. This is virtually identical to the current study. This was despite wide mean limits of agreement (range $-11.5 \%$ to $11.7 \%$ in the best study to $-15.4 \%$ to $26.2 \%$ in the worst study, with an overall average of $-16 \%$ to $18 \%$ ). In the combined data from the current study and the study of Naik and colleagues, ${ }^{30}$ only two of 260 patients $(0.8 \%, 0.1 \%$ to $2.8 \%)$ would have had differences of major clinical significance between the two techniques, validating echocardiography further. 


\section{Conclusions}

Thus, echocardiography with Simpson's apical biplane method is a valid tool to screen for LVSD in chronic survivors of AMI, finding few differences of major clinical significance despite relatively wide $95 \%$ limits of agreement and a high prevalence of significant regional wall motion abnormalities. This study therefore supports the use of echocardiography in screening for LVSD in chronic survivors of AMI or alternative high risk patients, with few errors of major clinical significance likely to occur.

\section{Authors' affiliations}

G I W Galasko, A Lahiri, R Senior, Department of Cardiovascular Medicine, Northwick Park Hospital, Harrow, Middlesex, UK S Basu, Department of Cardiology, Heatherwood and Wexham Park Hospital, London Road, Ascot, Berkshire, UK

\section{REFERENCES}

1 McMurray JJV, Petrie MC, Murdoch DR, et al. Clinical epidemiology of heart failure: public and private health burden. Eur Heart $J$ 1998; 19(suppl P):P9-16.

2 McMurray JV, McDonagh TA, Davie AP, et al. Should we screen for asymptomatic left ventricular dysfunction to prevent heart failure? Eur Heart $J$ $1998 ; 19: 842-6$

3 Tsutamoto T, Wada A, Maeda K, et al. Plasma brain natriuretic peptide level as a biochemical marker of morbidity and mortality in patients with asymptomatic or minimally symptomatic left ventricular dysfunction. Eur Heart $J$ 1999;20:1799-807

4 Anon. Effects of enalapril on mortality and the development of heart failure in asymptomatic patients with reduced left ventricular ejection fractions. The SOLVD investigators. N Engl J Med 1992;327:685-91.

5 Cleland JGF, Swedberg K, Poole-Wilson PA. Successes and failures of current treatment of heart failure. Lancet 1998;352(suppl 1):19-28.

6 Remes J, Miettinen H, Reunanen A, et al. Validity of clinical diagnosis of heart failure in primary health care. Eur Heart $J 1991 ; 12: 315-21$.

7 Williams JF, Bristow MR, Fowler MB, et al. Guidelines for the evaluation and management of heart failure. Report of the American College of Cardiology/ American Heart Association Task Force on Practice Guidelines (Committee on Evaluation and Management of Heart Failure). J Am Coll Cardiol 1995;26:1376-98.

8 Mosterd A, de Bruijne MC, Hoes AW, et al. Usefulness of echocardiography in detecting left ventricular dysfunction in population-based studies (the Rotterdam study). Am J Cardiol 1997;79:103-4.

9 Schiller NB, Shah PM, Crawford M, et al. Recommendations for quantitation of the left ventricle by two-dimensional echocardiography. American Society of Echocardiography Committee on Standards, Subcommittee on Quantitation of Two-Dimensional Echocardiograms. J Am Soc Echocardiogr 1989;2:358-67.

10 McDonagh TA, Morrison CE, Lawrence A, et al. Symptomatic and asymptomatic left ventricular systolic dysfunction in an urban population. Lancet 1997;350:829-33

11 Redfield MM, Jacobsen SJ, Burnett JC, et al. Burden of systolic and diastolic ventricular dysfunction in the community: appreciating the scope of the heart failure epidemic. JAMA 2003;289:194-202.

12 Galasko GIW, Lahiri A, Senior R. Portable echocardiography: an innovative tool in screening for cardiac abnormalities in the community Eur J Echocardiogr 2003;4:119-27.
13 Bellenger NG, Burgess MI, Ray SG, et al. Comparison of left ventricular ejection fraction and volumes in heart failure by echocardiography, radionuclide ventriculography and cardiovascular magnetic resonance: are they interchangeable? Eur Heart J 2000;21:1387-96.

14 Ray SG, Metcalfe MJ, Oldroyd KG, et al. Do radionuclide and echocardiographic techniques give a universal cut off value for left ventricular ejection fraction that can be used to select patients for treatment with ACE inhibitors after myocardial infarction. Br Heart J 1995;73:466-9.

15 Nicolosi GL, Latini R, Marino $P$, et al. The prognostic value of predischarge quantitative two-dimensional echocardiographic measurements and the effects of early lisinopril treatment on left ventricular structure and function after acute myocardial infarction in the GISSI-3 trial. Eur Heart $J$ 1996;17:1646-56.

16 Galasko GI, Basu S, Lahiri A, et al. A prospective comparison of echocardiographic wall motion score index and radionuclide ejection fraction in predicting outcome following acute myocardial infarction. Heart 2001;86:271-6.

17 Hellermann JP, Jacobsen SJ, Reeder GS, et al. Heart failure after myocardial infarction: prevalence of preserved left ventricular systolic function in the community. Am Heart J 2003;145:742-8.

18 Wackers FJ, Berger HJ, Johnstone DE, et al. Multiple gated cardiac blood pool imaging for left ventricular ejection fraction: validation of the technique and assessment of variability. Am J Cardiol 1979:43:1159-66.

19 Upton MT, Rerych SK, Newman GE, et al. The reproducibility of radionuclide angiographic measurements of left ventricular function in normal subjects at rest and during exercise. Circulation 1980;62:126-32.

20 van Royen N, Jaffe CC, Krumholz HM, et al. Comparison and reproducibility of visual echocardiographic and quantitative radionuclide left ventricular ejection fractions. Am J Cardiol 1996;77:843-50.

21 Basu S, Senior R, Raval U, et al. Beneficial effects of intravenous and oral carvedilol treatment in acute myocardial infarction: a placebo-controlled, randomized trial. Circulation 1997;96:183-91.

22 Haines AD, Khawaja IA, Hinge DA, et al. Radionuclide left ventricular ejection fraction: a comparison of three methods. Br Heart J 1987;57:242-6.

23 Senior R, Sridhara BS, Basu S, et al. Comparison of radionuclide ventriculography and $2 \mathrm{D}$ echocardiography for the measurement of leff ventricular ejection fraction following acute myocardial infarction. Eur Heart $J$ 1994:15:1235-9.

24 Starling MR, Crawford MH, Sorenson SG, et al. Comparative accuracy of apical biplane cross-sectional echocardiography and gated equilibrium radionuclide angiography for estimating left ventricular size and performance. Circulation 1981;63:1075-84.

25 Senior R, Basu S, Khattar R, et al. Independent prognostic value of the extent and severity of systolic wall thickening abnormality at infarct site after thrombolytic therapy. Am Heart J 1998;135:1093-8.

26 Vasan RS, Benjamin EJ, Larson MG, et al. Plasma natriuretic peptides for community screening for left ventricular hypertrophy and systolic dysfunction. The Framingham heart study. JAMA 2002;288:1252-9.

27 Fleiss JL. Statistical methods for rates and proportions, 2nd edn. New York: Wiley, 1981:218

28 Urena PE, Gervasio AL, Mitchell G, et al. Ejection fraction by radionuclide ventriculography and contrast left ventriculogram. A tale of two techniques. J Am Coll Cardiol 1999:33:180-5.

29 Schneider RM, Jaszczak RJ, Coleman RE, et al. Disproportionate effects of regional hypokinesis on radionuclide ejection fraction: compensation using attenuation-corrected ventricular volumes. J Nucl Med 1984:25:747-54.

30 Naik MM, Diamond GA, Soffer A, et al. Correspondence of left ventricular ejection fraction determinations from two-dimensional echocardiography, radionuclide ventriculography and contrast cineangiography. J Am Coll Cardiol 1995;25:937-42

31 Jensen-Urstad K, Bouvier F, Hojer J, et al. Comparison of different echocardiographic methods with radionuclide imaging for measuring left ventricular ejection fraction during acute myocardial infarction treated by thrombolytic therapy. Am J Cardiol 1998;81:538-44. 